\title{
STUDY OF ARRAY BI-CONICAL ANTENNA FOR DME APPLICATIONS
}

\author{
Ouadiaa BARROU ${ }^{1}$, Abdelkebir EL AMRI ${ }^{2}$ and Abdelati REHA ${ }^{3}$ \\ ${ }^{1}$ RITM Laboratory, CED Engineering Sciences, ESTC, Hassan II University of \\ Casablanca, Morocco \\ ${ }^{2}$ RITM Laboratory, CED Engineering Sciences, ESTC, Hassan II University of \\ Casablanca, Morocco \\ ${ }^{3}$ Laboratoire d'Innovation en Management et en Ingénierie pour l'Entreprise (LIMIE) \\ ISGA
}

\begin{abstract}
This paper introduces a new configuration of array bi-conical antenna to enhance the gain of an antenna for Distance Measuring Equipment (DME) avionic system. Due to its large size, the antenna can be placed in terrestrials DME stations. The antenna consists of the bi-conical elements placed in a linear configuration. The simulated maximum gain is $10.2 \mathrm{~dB}$, the antenna operates in the DME band (960$1215 \mathrm{MHz}$ ). Al the simulations are performed with CADFEKO a Method of Moments based Solver.
\end{abstract}

\section{KEYWORDS}

DME, Array antennas, Bi-conical Antennas

\section{INTRODUCTION}

Distance Measuring Equipment (DME) is a Radio Navigation technology that measures the slant range (distance) between an aircraft and a ground station by timing the propagation delay of radio signals in the frequency band between 960 and $1215 \mathrm{MHz}$. Line-of-visibility between the aircraft and ground station is required. An interrogator (airborne) initiates an exchange by transmitting a pulse pair, on an assigned 'channel', to the transponder ground station. The channel assignment specifies the carrier frequency and the spacing between the pulses. After a known delay, the transponder replies by transmitting a pulse pair on a frequency that is offset from the interrogation frequency by $63 \mathrm{MHz}$ and having specified separation. The DME consists of two parts, the first one is embedded on the aircraft and the other is installed in ground stations [1-5]. The design of a DME system encounters several challenges among which: the development of signal analysis algorithms for signals coming from aircrafts and the design of antennas with very high gains in order to cover a long range. The purpose of this paper is the design of an operational array antenna for DME ground stations.

\section{Distance Measuring Equipment Presentation}

DME is an international, standardized navigation system. It allows an aircraft to automatically measure its physical line of sight distance (in nautical miles) from a selected ground-based beacon (Figure 1).

DME systems are used throughout the world by all airliners, most military aircraft, and a large number of general-aviation aircraft. The range of service is most often up to 300 land miles (480 
$\mathrm{km})$. System accuracy is usually 0.1 nautical miles $(185 \mathrm{~m})$ but precision equipment, intended for use during landing, has accuracy up to $100 \mathrm{ft}(30 \mathrm{~m})$. The airborne equipment, called an interrogator, transmits pulses of $1 \mathrm{~kW}$ peak power on 1 of 126 frequencies. These frequencies are in the $1025-1150-\mathrm{MHz}$ band and are spaced $1 \mathrm{MHz}$ apart (Figure 2). Each pulse has a duration of $3.5 \mu \mathrm{s}$ and is paired with another, spaced $12 \mu \mathrm{s}$ (X Mode) or $36 \mu \mathrm{s}$ (Y Mode) (i.e. "delayed" by either 12 or $36 \mu$ s) (Figure 3).

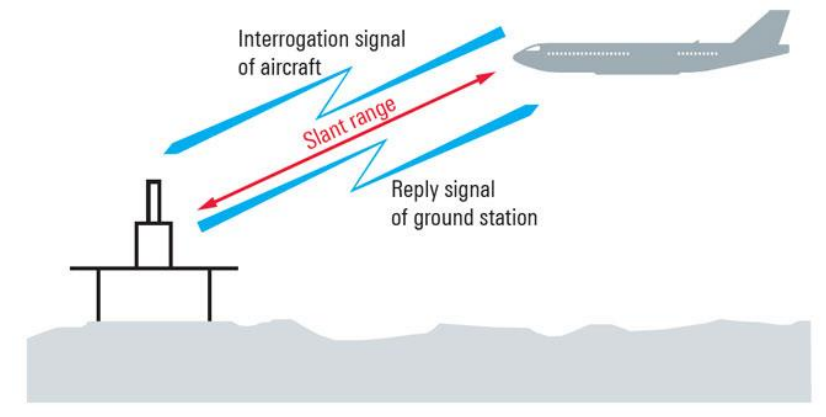

Figure 1. DME System
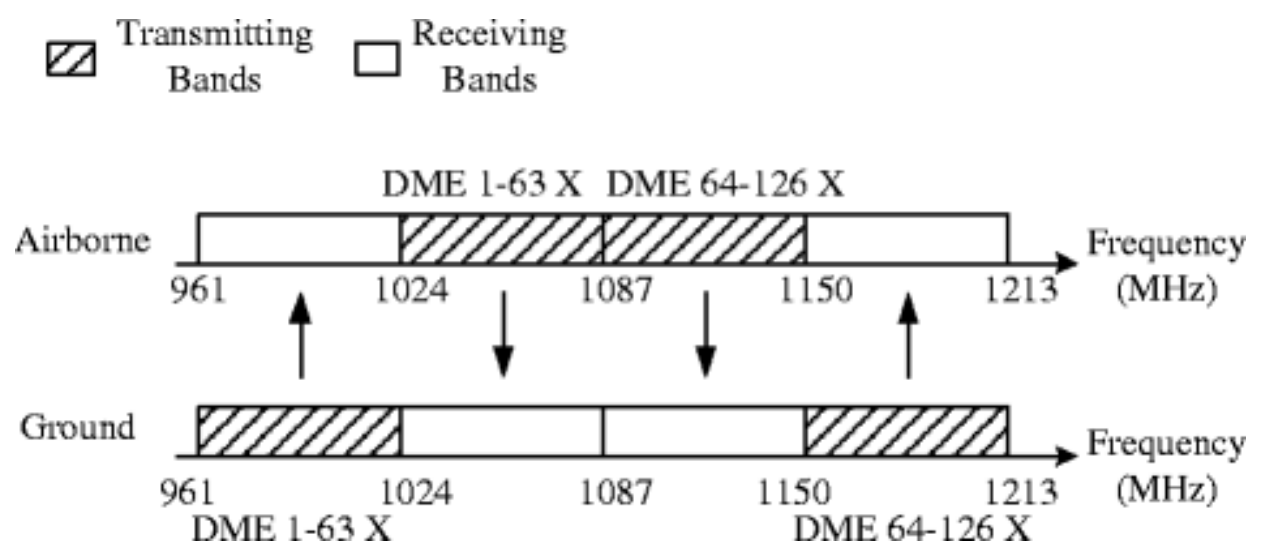

Figure 2. Frequency band and channels

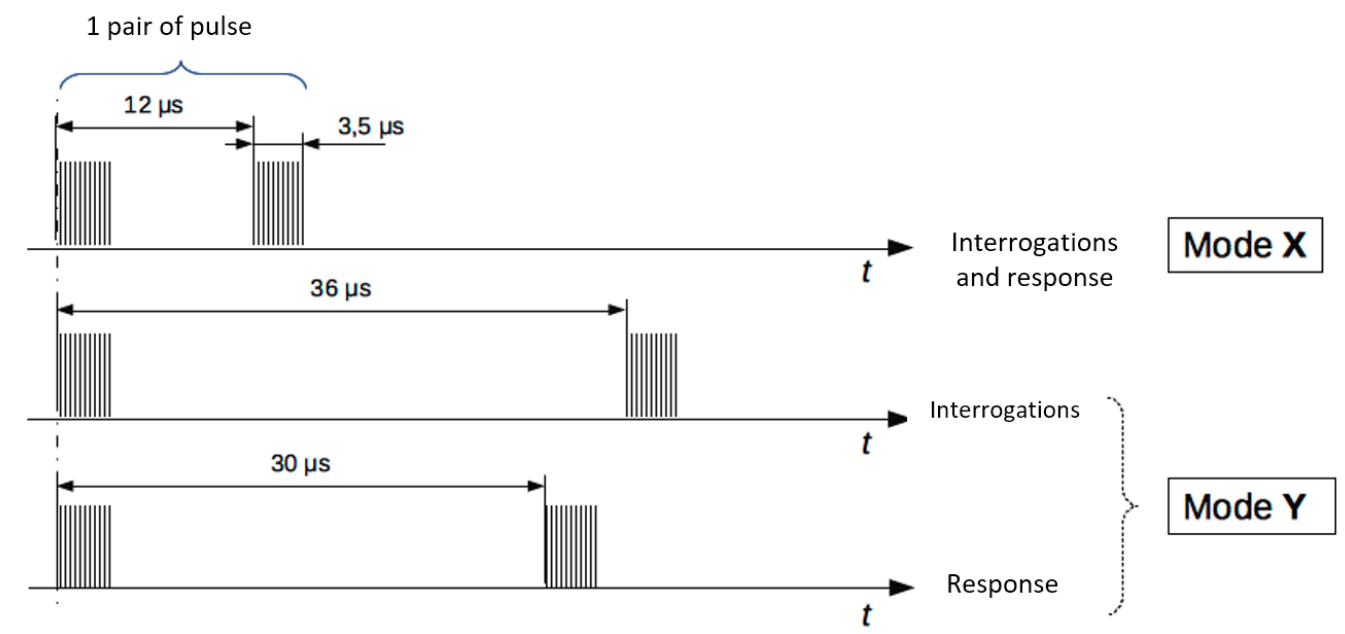

Figure 3. The two DME Modes X and Y

The combination of frequencies and pulse spacing therefore provides 252 operating channels in the system. The beacon equipment on the ground is called a transponder. It receives these 
pulses, delays them by $50 \mu \mathrm{s}$, and then retransmits them, usually with a power of $1 \mathrm{~kW}$, on 252 frequencies lying between 962 and $1213 \mathrm{MHz}$. The pulse-pair spacing is $12 \mu \mathrm{s}$ on those frequencies not used by the interrogator (X Mode), and $36 \mu$ s on those frequencies shared with the interrogator (Y Mode) (Figure 3). The transponder transmission is called the reply. The frequency difference between interrogation and reply is always $63 \mathrm{MHz}$. This arrangement allows each transmitter frequency to act as the local oscillator for its associated receiver, the intermediate frequency of which is $63 \mathrm{MHz}$. For landing purposes, some transponders have powers as low as $100 \mathrm{~W}$. In the aircraft the replies to its own interrogations are recognized by their phase coherence with their own transmissions, and by the elapsed time measured between transmissions and reception (minus the $50 \mu$ s transponder delay), usually by means of a crystal clock. This elapsed time is about $12.36 \mu$ s for each nautical mile, and the measured distance is displayed in the cockpit on a digital meter, which is usually calibrated in nautical miles and tenths of nautical miles (Figure 4). We can find one or more DME system in an airplane [1-5].

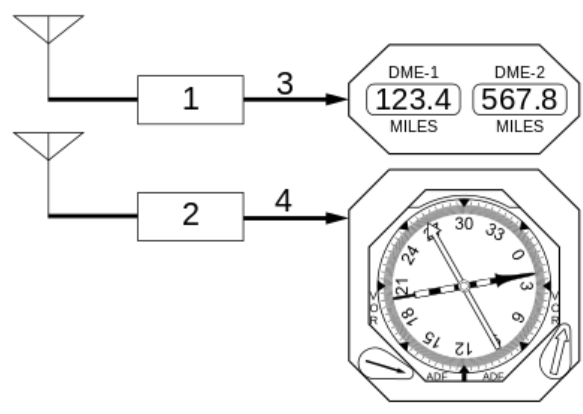

Figure 4. DME meter

\section{Bi-Conical Antenna Analysis}

The bi-conical antennas can be divided into two types according to their cone length (l), one is infinite length bi-conical antenna, and another is finite one, as illustrated in Figure 5. When the half-cone angles $\theta_{1}=\theta_{2}$, the bi-conical antenna is called symmetrical bi-conical, otherwise the antenna is asymmetrical bi-conical [6-8].
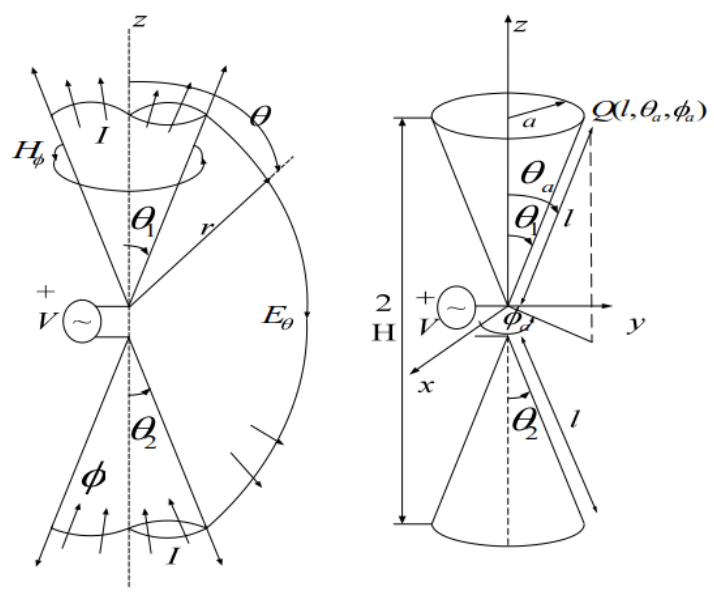

Figure 5. Infinite length and finite length bi-conical antenna [2-3]

The bi-conical antenna is most readily handled in spherical coordinates. If the dominant fields are satisfied for a spherical TEM mode condition with respect to the r-direction, the fields $\mathrm{E}_{\theta}$ and $\mathrm{H}_{\varphi}$ in the infinite bi-conical horn antenna are: 
International Journal of Wireless \& Mobile Networks (IJWMN) Vol. 11, No. 4, August 2019

$$
\begin{gathered}
E_{\theta}=\frac{V_{0} e^{-j k r}}{r \sin \theta \cdot \ln \left\{\left(\cot \frac{\theta_{1}}{2}\right)\left(\cot \frac{\theta_{2}}{2}\right)\right\}} \\
H_{\varphi}=\frac{V_{0} e^{-j k r}}{\eta \cdot r \cdot \sin \theta \cdot \ln \left\{\left(\cot \frac{\theta_{1}}{2}\right)\left(\cot \frac{\theta_{2}}{2}\right)\right\}}
\end{gathered}
$$

At point $\mathrm{Q}\left(1, \theta_{\mathrm{a}}, \varphi_{\mathrm{a}}\right)$, when driving voltage is $\mathrm{V}_{0}$, from the equivalent Huygens source and the wavefront field given by (l), the radiation field $\mathrm{dE}_{\theta}$ of the infinitesimal aperture is :

$$
\begin{aligned}
d E_{\theta}= & \frac{-j k l^{2} e^{-j k r}}{4 \pi r} \\
& \cdot \exp \left\{-j k l\left[\sin \theta \sin \theta_{a} \cos \left(\phi-\phi_{a}\right)+\cos \cos \theta_{a}\right]\right. \\
& \cdot E_{\theta_{1}}\left\{\sin \theta \sin ^{2} \theta_{a}+\cos \theta \cos \theta_{a} \sin \theta_{a} \cos \left(\phi-\phi_{a}\right)\right\} \\
& +\sin \theta_{a} \cos \left(\phi-\phi_{a}\right) d \theta_{a} d \phi_{a}
\end{aligned}
$$

Where the field $\mathrm{E}_{\theta 1}$, is the same field as the infinite bi-conical horn antenna at the cone length $l$ (along the surface), and the $\mathrm{E}_{\theta 1}$ is from (1)

$$
E_{\theta_{1}}=\frac{V_{0} e^{-j k l}}{l \sin \theta \cdot \ln \left\{\left(\cot \frac{\theta_{1}}{2}\right)\left(\cot \frac{\theta_{2}}{2}\right)\right\}} .
$$

Therefore, radiation field $E_{\theta}$ of the asymmetrical bi-conical horn antenna from (2) and (3) reduces to

$$
\begin{aligned}
E_{\theta}= & \int_{0}^{2 \pi} d \phi_{a} \int_{\theta_{1}}^{\pi-\theta_{2}} d E_{\theta} d \theta_{a} \\
& \frac{j k l V_{0} e^{-j k(r+l)}}{2 \cdot r \cdot \ln \left\{\left(\cot \frac{\theta_{1}}{2}\right)\left(\cot \frac{\theta_{2}}{2}\right)\right\}} \cdot\left[\int_{\theta_{1}}^{\pi-\theta_{2}} e^{j k l \cdot \cos \theta \cdot \cos \theta_{a}}\right. \\
& \cdot\left\{\sin \theta \sin \theta_{a} J_{0}\left(k l \sin \theta \sin \theta_{a}\right)\right. \\
& \left.\left.+j\left(1+\cos \theta \cos \theta_{a}\right) J_{1}\left(k a \sin \theta \sin \theta_{a}\right)\right\} d \theta_{a}\right]
\end{aligned}
$$

Where $J_{n}(n=0,1)$ is first kind of Bessel function.

By numerically integrating over the antenna aperture, the radiation patterns are obtained for an asymmetrical bi-conical horn antenna. The total field of the array is determined by the vector sum of the fields radiated by the individual elements.

The input impedance of single finite length symmetry bi-conical antenna is [5] 


$$
Z_{0}=\frac{\eta}{\pi} \ln \left(\cot \frac{\theta_{1}}{2}\right)
$$

In addition, the input impedance of single finite length asymmetry bi-conical antenna is [5]

$$
Z_{1}=\frac{\eta}{2 \pi} \ln \left[\left(\cot \frac{\theta_{1}}{2}\right)\left(\cot \frac{\theta_{2}}{2}\right)\right]
$$

Where $\eta$ is transmission medium impedance, and when medium is free space, $\eta=367.7 \Omega \approx 120 \pi \Omega$.

The total antenna array impedance at a driving point is the parallel combination of terminal impedances as described in (7).

$$
Z_{\text {array }}=\frac{1}{\frac{1}{Z_{1}}+\frac{1}{Z_{2}}+\frac{1}{Z_{3}}+\ldots+\frac{1}{Z_{N}}}
$$

Where $\mathrm{N}$ is the number of bi-conical linear array element. All the impedance in this paper is set according to (8).

\section{DESIGN OF LINEAR ARRAY BI-CONICAL ANTENNA}

\subsection{The single element bi-conical antenna}

As shown in figure 6, the single element bi-conical antenna is composed of two cones each one have a height $(\mathrm{H})$ and a radius $(\mathrm{R})$. (Figure 6). After design and optimisation with CADFEKO a Method of Moments (MoM) based solver [14], the values of $H=44 \mathrm{~mm}$ and $\mathrm{R}=15 \mathrm{~mm}$. As shown in figure 7, the antenna operates in the band of $960-1215 \mathrm{MHz}$ which can make it an adequate solution for the DME system. But as show in figure 7(b), the gain is not big enough.

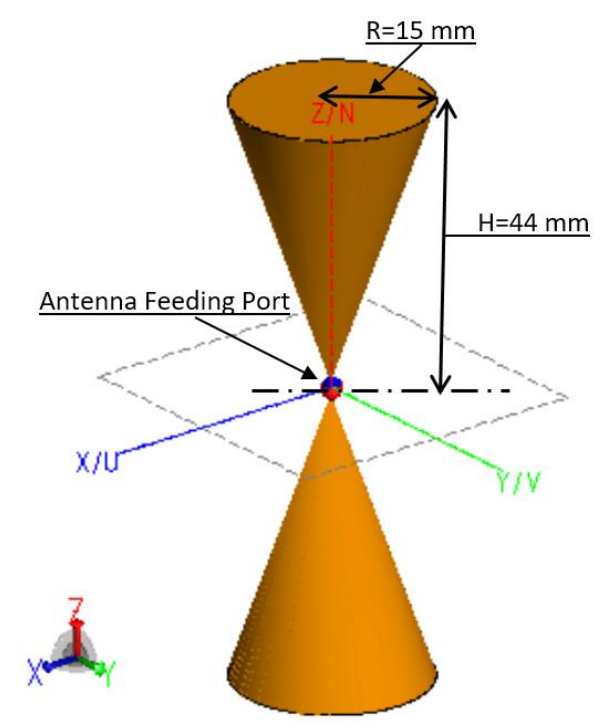

Figure 6. The geometry of single bi-conical antenna 


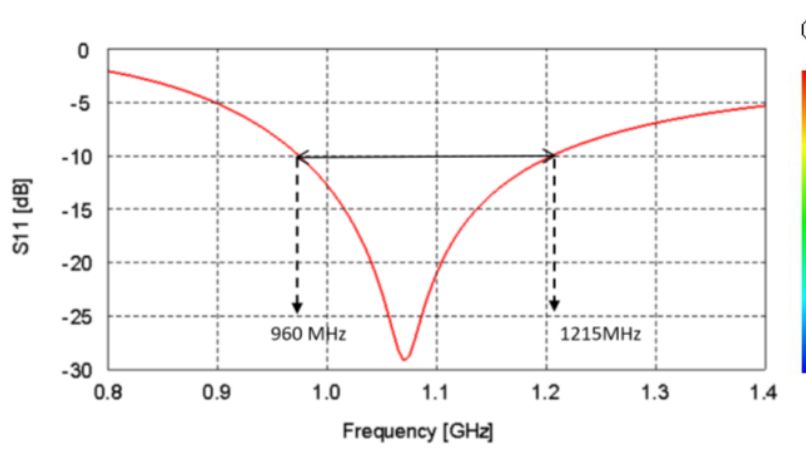

(a) $\mathrm{S}_{11}$ parameter

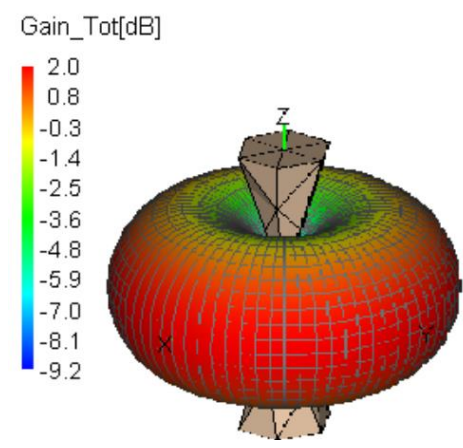

(b) 3D gain pattern

Figure 7. The Simulated $S_{11}$ parameter (a) and the simulated 3D gain pattern of single bi-conical antenna (b)

\subsection{Two elements bi-conical antenna}

After optimisation with CADFEKO, the two elements of bi-conical antenna is composed of two bi-conical elements separated by $\mathrm{D}=0.75 * \lambda=204 \mathrm{~mm}$ ( $\lambda$ for the resonance frequency $\mathrm{f}=1.07 \mathrm{GHz}$ ) (Figure 8). Figure 9 shows the $S_{11}$ and $S_{21}$ parameters versus frequencies, the antenna operates in the band of $960-1215 \mathrm{MHz}$ which can make it an adequate solution for the DME system. The $S_{22}$ and $S_{12}$ parameters are respectively the same as the $S_{11}$ and $S_{21}$ parameters. We observe that the gain increases from $2 \mathrm{~dB}$ to $2.9 \mathrm{~dB}$.

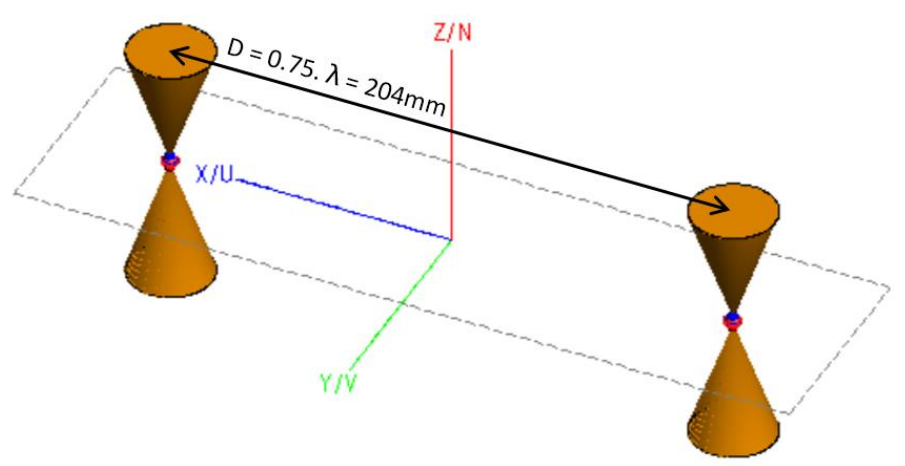

Figure 8. The geometry of two elements bi-conical antenna

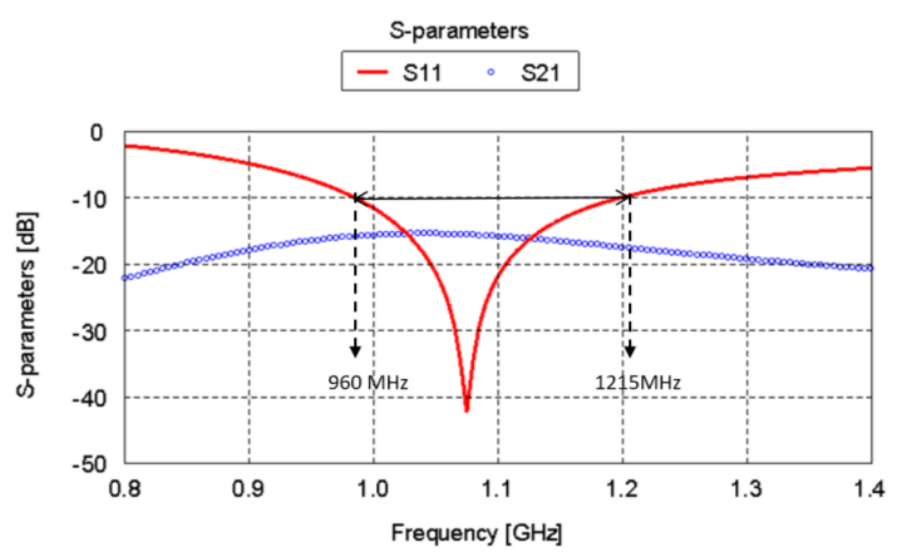

(a) $S_{11}$ parameter

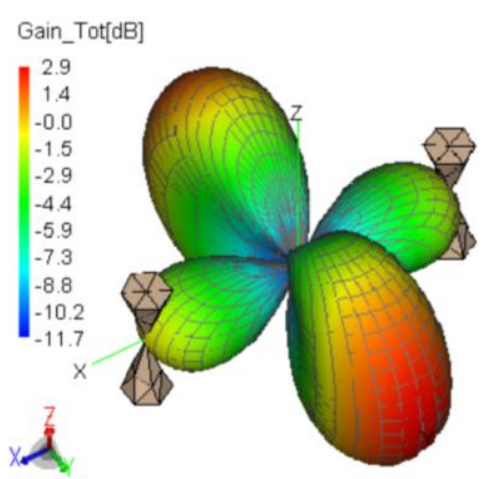

(b) 3D gain pattern

Figure 9. The Simulated $S_{11}$ parameter (a) and the simulated 3D gain pattern of two elements bi-conical antenna (b) 


\subsection{Four, eight and ten elements bi-conical antenna}

When we increase the number of elements, we observe that the gain increases. For the four, eight and ten elements antenna the maximum gains are respectively $6.2 \mathrm{~dB}, 9.3 \mathrm{~dB}$ and $10.2 \mathrm{~dB}$. The main lobe becomes very narrow so the antenna becomes more directive (figure 10).

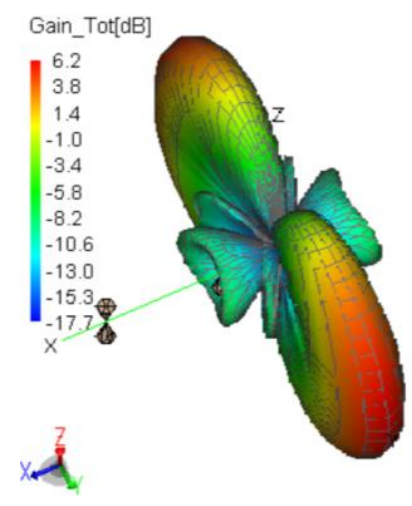

(a) Four elements

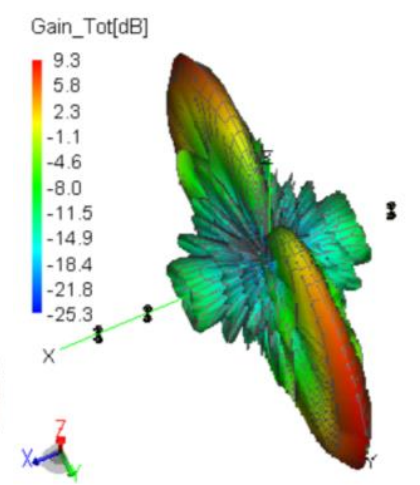

(b) Eight elements

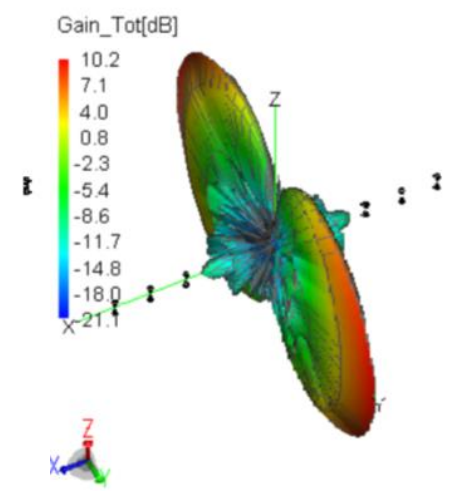

(c) Ten elements

Figure 10. The simulated 3D gain pattern of four (a), eight (b) and ten (c) elements bi-conical antenna at the resonance frequency $(1.07 \mathrm{GHz})$

\section{COMPARISON WITH OTHER WORKS}

The comparison with the results found by Qiuyuan Pang and Yude Ni [6][11] shows that this configuration allows to obtain a higher gain $10.2 \mathrm{~dB}$ for 10 elements versus $9.38 \mathrm{~dB}$ for the same number of elements. The difference between the two works is: in [6][11], the ten elements are place as shown in figure 11(a), but in our work these elements are placed as shown in figure 11(b).

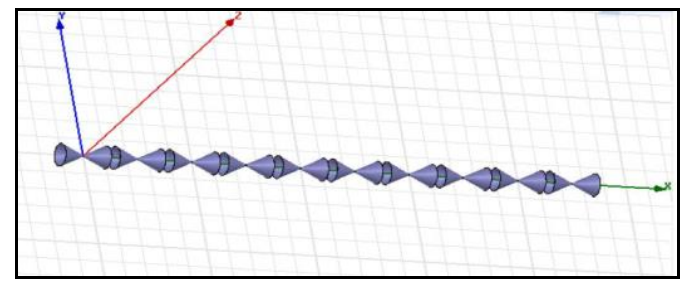

(a) Qiuyuan Pang work [6][11]

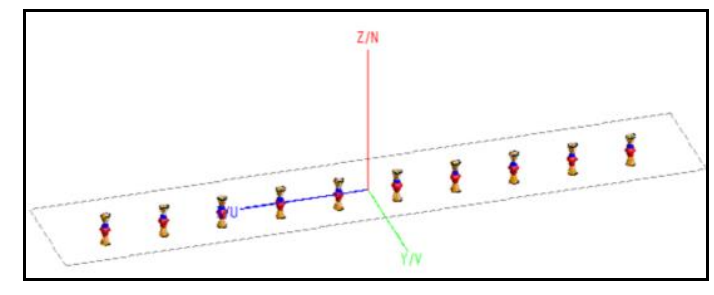

(b) our work

Figure 11. The comparison between Qiuyuan Pang work (a) and our work (b)

In 2014, Amna Ikram et Al, studied a Low Profile Aircraft Antenna with gains less than 4dB [9]. Mabrouk designed an inverted L-shaped antenna with omni directional gain pattern and the gain that varies between 2 and $5 \mathrm{~dB}[12]$.

In 2016, Icev has studied the state of the art of embedded antennas on aircrafts. All the studied antennas are miniaturized and have the maximum gains varied between 17 and $80 \mathrm{~dB}$ [10]. Bhavya designed a Conformal Printed sleeve dipole antenna operating in $800 \mathrm{MHz}$ to $3000 \mathrm{MHz}$ with gains between 0.4 and $2.26 \mathrm{~dB}$ [13]. 


\section{CONCLUSION}

A new configuration of array bi-conical antenna to enhance the gain of an antenna for DME avionic system has been studied. The antenna operates in the DME band $(960-1215 \mathrm{MHz})$, the simulated maximum gain reaches the value of $10.2 \mathrm{~dB}$. Due to its large size, the antenna can be placed in terrestrials DME stations.

In the next work, fabrication and measurement should be done to confirm the simulated results. Also, more refinements should be done to enhance the gain of the antenna.

\section{REFERENCES}

[1] Federal Aviation Administration, Pilot's Handbook of Aeronautical Knowledge, FAA, U.S. Department of Transportation, 2016

[2] Annex 10 to the Convention on International Civil Aviation, Volume I - Radio Navigation Aids; International Civil Aviation Organization; International Standards and Recommended Practices, www (.) icao (.) int

[3] Borje Forssell, Radionavigation Systems, Artech House Publishers, february 2008

[4] Federal Aviation Administration, Aeronautical Chart User's Guide, Skyhorse, 2017

[5] Cary Spitzer et Al, Digital Avionics Handbook, CRC Press, 2014

[6] Qiuyuan Pang and Yude Ni, Design and Radiation Characteristics Analysis of DME Beacon Antenna, Communications in Information Science and Management Engineering (CISME) Vol. 2 Iss. 42012 PP. 49-52

[7] Warren L. Stutzman, Gary A, Antenna Theory and Design, John Wiley \& Sons, 2012

[8] C.Balanis, Antenna Theory: Analysis and Design, Wiley-Interscience; 2005

[9] Amna Ikram, Jean-Jacques Laurin, On the Increase of the Directivity of a Low Profile Aircraft Antenna, The 8th European Conference on Antennas and Propagation, 2014

[10] Ilcev St. D, Airborne Satellite Navigation and Other Integrated Antenna Systems, International Conference on Electrical, Electronics, and Optimization Techniques (ICEEOT), 2016

[11] Yude Ni, Qiuyuan Pang, Zongping Lv, Far Field Radiation Characteristics of Linear Biconical Antenna Array, 2nd International Conference on Artificial Intelligence, Management Science and Electronic Commerce (AIMSEC), August 2011

[12] A Mabrook Masoud, Su Donglin, A novel wide-band airborne UHF antenna, IEEE Workshop on Electronics, Computer and Applications, June 2014.

[13] P. Bhavya et Al, Design and Development of a Conformal Printed sleeve dipole antenna operating in $800 \mathrm{MHz}$ to $3000 \mathrm{MHz}$, Indian Journal of Science and Technology, December 2016

[14] Atef Z. Elsherbeni, Payam Nayeri, C.J. Reddy, Antenna Analysis and Design using FEKO Electromagnetic Simulation Software, The ACES Series on Computational Electromagnetics and Engineering (CEME), SciTech Publishing, 2014 